\title{
Peritoneal Carcinomatosis Secondary to Papillary Renal Cell Carcinoma: Case Report
}

\author{
Aubanell A* | Mast R | Roche S | Semidey M | Suarez C | Trilla E \\ *Correspondence: Anton Aubanell
}

Address: Hospital Universitari Vall d'Hebron Barcelona, Spain

e-mail $\bowtie$ : ton.aubanell@gmail.com

Received: 25 March 2021; Accepted: 30 March 2021

Copyright: (C) 2021 Aubanell A. This is an open-access article distributed under the terms of the Creative Commons Attribution License, which permits unrestricted use, distribution, and reproduction in any medium, provided that the original work is properly cited.

\section{ABSTRACT}

Renal cell carcinoma accounts for approximately $5 \%$ and $3 \%$ of all neoplasms diagnosed in men and women respectively, making it the 7 th most common neoplasm in the adult population. Nowadays, localized neoplasm can be surgically treated and has a good overall prognosis. When presenting metastatic disease, the most frequent locations of metastasis are the lungs, liver, and bone. Approximately one third of patients with renal stage IV renal cell carcinoma will develop peritoneal metastatic disease but this is a rare presentation as first metastatic localization. We are presenting a 68-year-old patients who underwent partial nephrectomy to treat a localized renal carcinoma who three years after surgery presented peritoneal carcinomatosis as first metastatic location.

Keywords: Renal Cell Carcinoma, Peritoneal Carcinomatosis, Biopsy, CT-Scan, PET-CT

\section{Introduction}

Renal cell carcinoma accounts for approximately $5 \%$ and $3 \%$ of all neoplasms diagnosed in males and females respectively, making it the 7th most common neoplasm in the adult (Escudier et al., 2019)

The classical presentation of this entity consists of the following triad (Cohen and Mcgovern, 2005): pain on the affected flank, hematuria, and the palpable mass. Currently, this is a rare presentation, being more commonly found as an incidental finding in an imaging test (Cohen and Mcgovern, 2005).

Renal cancer is classified is four main groups according to its anatomopathological characteristics (Cohen and Mcgovern, 2005): clear cell carcinoma (75\%), papillary carcinoma (12\%), chromophobe carcinoma (4\%) and oncocytoma (4\%).

We are presenting a case of a patient with renal papillary carcinoma, an entity with a prevalence for the male sex (5:1). Papillary carcinoma can be classified in two subgroups (Escudier et al., 2019): type I and type II being the latter the one with worse prognosis and the greatest incidence of recurrences (Escudier et al., 2019). Although the prevalence of metastasis is more common in clear cell carcinoma 
than in papillary, the latter group has a worse prognosis when metastasis are present (Cohen and Mcgovern, 2005).

Peritoneal involvement of renal neoplasm is a rare complication that has not been systematically studied due to the low incidence of this complication. One cohort has reported incidences up to $20 \%$ during the evolution of the disease with an average time of 16 months between diagnosis of metastatic disease and the development of carcinomatous ascites (Sidana et al., 2017).

In our case the patient had peritoneal carcinomatosis as the first relapse of a kidney neoplasm intervened by laparoscopic partial nephrectomy. The special interest in this case lies in the infrequent presentation of this entity being peritoneal metastasis the first remote manifestation of a retroperitoneal neoplasm.

\section{Clinical Presentation}

We are presenting a 68-year-old male patient, active smoker. This patient had been diagnosed with a renal papillary type II adenocarcinoma at the inferior pole of $6 \mathrm{~cm}$ diameter (T1B, N0, M0) which was treated by laparoscopic partial nephrectomy three years before the present time. The patient had no other pathological history relevant to the case.

The patient showed no new signs of disease recurrence until a control CT study conducted three years after the intervention. In this abdominal CT study, performed with no iodate contrast due to the antecedent of an allergic reaction, we found reticulation of the peritoneal fat accompanied by multiple micronodules. This presentation is suggestive of peritoneal carcinomatosis and is classically defined as "omental cake" (Fig. 1) (Zamir et al., 2011). No other solid lesions or lymphadenopathies were found in the CT scan.

With these findings and taking into consideration the history of neoplastic disease a nuclear medicine test was performed in order to complete the study and assess possible neoplastic lesions not visualized in the abdominal CT study.

A PET/CT study was conducted which found no other lesions suspicious of malignancy. The only positive finding in this test was the radiopharmaceutical deposit in the peritoneal cavity especially in prehepatic, left sub-phrenic and left para-colonic spaces droplet regions (Fig. 2). With these findings a differential diagnosis was established between relapse of the previous renal neoplasm, a new metastatic malignancy and a rare presentation of an infectious disease such as peritoneal tuberculosis (Zamir et al., 2011). 

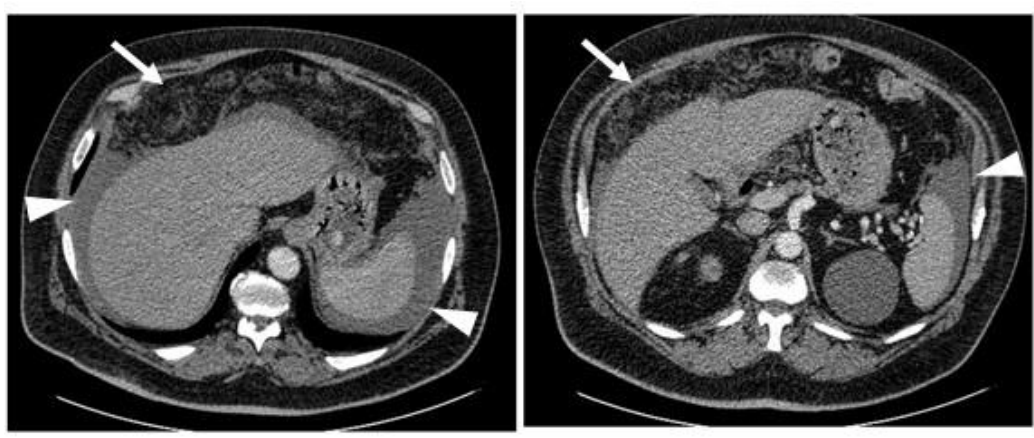

Figure 1: No-contrast enhanced CT-scan that shows peritoneal fat reticulation and micronodules, known as omental cake (arrow) and abdominal free liquid (arrowheads) suggesting peritoneal carcinomatosis

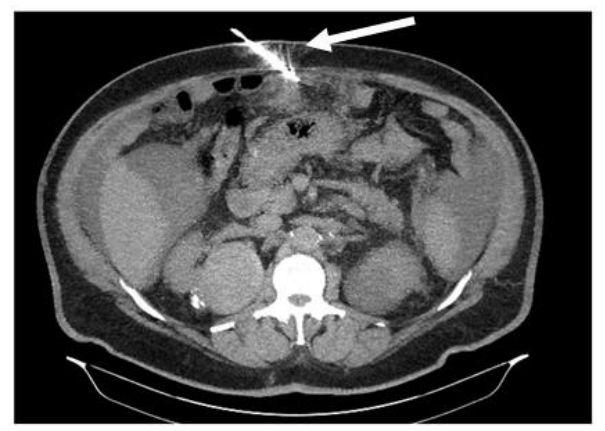

Figure 2: CT-scan guided biopsy of the peritoneal fat (arrow)

During the study, the patient developed a first event of ascites from which was obtaining a sample of ascitic fluid by paracentesis. The immunohistochemical profile of ascitic fluid sample cells matches that of the primary renal tumor operated three years earlier establishing a diagnostic suspicion of carcinomatosis ascites.

Being this a very unusual presentation, we decided to perform a biopsy of the peritoneal fat guided by CT (Fig. 3). The anatomopathological analysis of this biopsy was found compatible with papillary adenocarcinoma type II sharing immunohistochemical markers (EMA, VIM, CK7, PAX8) with those of the primary tumoral lesion (Table 1).

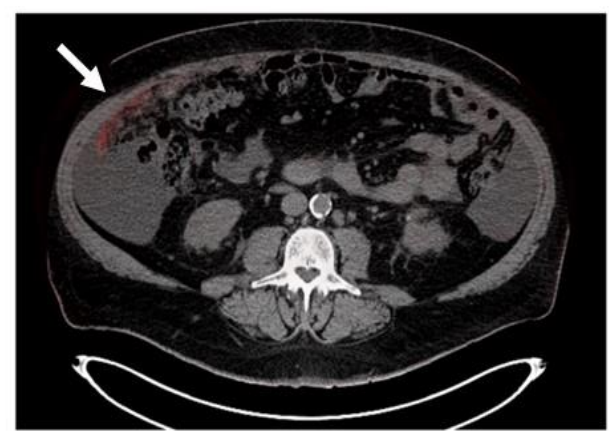

Figure 3: PET/CT scan with radiopharmaceutical deposit in the peritoneal cavity especially, pre-hepatic space (arrow) 
Table 1: Similarities between the primary renal tumor and the ascitic fluid immunohistochemical profiles

\begin{tabular}{|l|l|l|l|l|l|l|l|l|l|l|}
\hline & EMA & VIM & CK7 & CD10 & TFE3 & P504s & CD57 & WT1 & PAX8 \\
\hline Primary & + & + & + & + & - & + & + & - & + \\
\hline Biopsy & + & + & + & & - & & & & + \\
\hline
\end{tabular}

Diagnostic confirmation of peritoneal metastatic disease led to a re-staging of the neoplasia to a stage IV. With this diagnosis, the patient started palliative treatment by atezolizumab + bevacizumab (Rini et al., 2019) persisting multiple relapses in ascites that required palliative evacuating paracentesis. In the present moment, the patient presents a stable disease with a good overall performance status.

\section{Discussion}

This case is relevant for the atypical presentation of metastatic lesions in renal carcinoma, being peritoneal fat, the only region affected by the metastatic disease. We should keep in mind that the metastatic main metastatic locations of renal carcinoma tend to affect solid organs following the characteristic pattern of systemic venous drainage being the most frequently affected organs the lung, liver and bone (Zamir et al., 2011).

Peritoneal metastasis and tumoral ascites are considered factors of poor prognosis of neoplastic disease (Sidana et al., 2017). Ovarian, colorectal and pancreatic carcinomas are the neoplasms that more commonly metastasize to the peritoneum (Sidana et al., 2017). Only 2\% of cases of peritoneal carcinomatosis are secondary to renal carcinomas (Zamir et al., 2011).

In the case of renal carcinoma, peritoneal spaces most often affected by peritoneal carcinomatosis and ascites are Douglas space, mesosigma, and both para-cholic spaces (García et al., 2006).

Stavropoulos et al proposed two possible mechanisms to explain peritoneal carcinomatosis spread in renal cancer, a retroperitoneal neoplasm. These authors suggested that the involvement of the renal capsule and Gerota's fascia predisposes to a local peritoneal involvement. When the patient develops ascites, these carcinomatous cells are distributed through the rest of the peritoneum. Another theory that explains the peritoneal metastatic spread of renal carcinoma is through hematic metastasis to omentum, mesentery and peritoneum causing peritoneal implants, when ascites appears this carcinomatous cells will be distributed through the peritoneum (Stavropoulos et al., 1995)

In our case the patient had a neoplastic lesion of $6 \mathrm{~cm}$ T1b that did not affect adjacent structures or compromised vascular structures, being this the reason to choose a partial nephrectomy as the first therapeutic approach. As it is a localized disease, the chances of contiguous and vascular invasion are less likely. 
In postoperative patients, as in our case, it has been proposed that implantation occurs secondary to the disruption of the planes that define the peritoneal and retroperitoneal cavities during the surgery, being this the most probable way that tumoral cells spread into the peritoneal cavity.

\section{Conclusion}

Peritoneal carcinomatosis is a rare presentation of first metastatic location of renal cell carcinoma and a sign of bad prognosis. Story of surgical intervention as well as the presence of bulky tumor are risk factors for peritoneal disease spreading.

\section{References}

Cohen HT and Mcgovern FJ. Renal-Cell Carcinoma. N engl J Med 2005; 353: 2477-2490.

Escudier B, Porta C, Schmidinger M, Rioux-Leclercq N, Bex A, Khoo V, Gruenvald V, Horwich A. Renal cell carcinoma: ESMO Clinical Practice Guidelines for diagnosis, treatment and follow-up. Ann Oncol Off J Eur Soc Med Oncol 2019; 30: 706-720.

García NR, Tello AMG, González LL, Mediero JMG, Cuesta JA, Sánchez AB. Carcinoma renal metastásico a peritoneo. A propósito de un caso. Arch Esp Urol 2006; 59: 919-922.

Rini BI, Powles T, Atkins MB, Escudier B, McDermott DF, Suarez C, Bracarda S, Stadler WM, Donskov F, Lee JL, Hawkins R, Ravaud A, Alekseev B, Staehler M, Uemura M, De Giorgi U, Mellado B, Porta C, Melichar B, Gurney H, Bedke J, Choueiri TK, Parnis F, Khaz MR. IMmotion151 Study Group. Atezolizumab plus bevacizumab versus sunitinib in patients with previously untreated metastatic renal cell carcinoma (IMmotion151): A multicentre, open-label, phase 3, randomised controlled trial. Lancet 2019; 393: 2404-2415.

Sidana A, Kadakia M, Friend JC, Krane LS, Su D, Merino MJ, Srinivasan R. Determinants and prognostic implications of malignant ascites in metastatic papillary renal cancer. Urol Oncol Semin Orig Investig 2017; 35: 114.e9-114.e14.

Stavropoulos NJ, Deliveliotis C, Kouroupakis D, Demonakou M, Kastriotis J, Dimopoulos C. Renal cell carcinoma presenting as a large abdominal mass with an extensive peritoneal metastasis. Urol Int 1995; 54: 169-170.

Zamir N, Akhtar J, Ahmed S. Omental cake: a radiological diagnostic sign. APSPJ Case Rep 2011; 2: 27. 